\title{
Cardiac sequelae of acute head injury
}

Sir,

The study of McLeod et al. ${ }^{1}$ on cardiac sequelae of acute head injury postulated that the cause of these changes was catecholamine excess. We would like to offer direct myocardial contusion as a more likely explanation.

The seven patients under study were victims of road accidents with head injuries. The mechanism and severity of injury are consistent with other, unrecognised disease. ${ }^{2}$ The type of decelerative force involved in most automobile accidents is commonly the cause of myocardial contusion. Visible chest wall trauma need not be present for significant myocardial injury to occur. ${ }^{3}$ Up to $70 \%$ of patients with myocardial contusion complain of precordial chest pain similar to that of myocardial ischaemia. ${ }^{4}$ The state of consciousness of these patients makes evaluation of these subjective complaints difficult.

With myocardial contusion, the most common finding on physical examination is tachycardia, unexplained by other causes. ${ }^{+}$Electrocardiographic changes include sinus tachycardia, ST segment abnormalities, $\mathrm{T}$ wave flattening or inversion, and cardiac arrhythmias. ${ }^{5}$

In this study, a heart rate of over 150 was reported in four patients and ST segment depression in three. Cardiac arrhythmias were also described. Elevation of the MB isoenzyme of creatine phosphokinase was noted in six. This is helpful in the diagnosis of myocardial contusion and, in conjunction with electrocardiographic abnormalities, has been found to be associated with a high frequency of complications. ${ }^{6} 7$ This pattern was seen in at least one of the fatalities reported in this study. Focal myocardial necrosis can be seen ${ }^{+}$and was found at necropsy in one of the fatalities in this series. It is difficult to attribute this to catecholamine excess unless prolonged coronary artery spasm or occlusion sufficient to cause myocardial infarction occurred. This would be unusual, especially with no previous history of cardiac disease.

It is postulated that tachycardia in myocardial contusion is secondary to decreased cardiac output from injured, poorly functioning myocardium. ${ }^{{ }}$It is possible that the pronounced tachycardia reported in these patients was sufficient to account for the normal cardiac outputs recorded. In this respect, tachycardia and increased catecholamine levels are appropriate compensatory responses. Sympathetic blockade, suggested as treatment, would be detrimental rather than beneficial, because it would eliminate these changes, thereby further compromising an already stressed myocardium.

The need for a high clinical suspicion to make the diagnosis of myocardial contusion is frequently stressed. ${ }^{+}$The suggestion that head trauma may cause these secondary cardiac changes is interesting. It must be studied, however, in cases of direct, isolated head injury to eliminate unrecognised direct cardiac injury, which may have played a role in the cases reported here.

Ted Larremore, Vince Markovchick, Emergency Medical Services, Denver General Hospital, 750 Cherokee Street, Denver, Colorado 80204, USA.

\section{References}

1 McLeod AA, Neil-Dwyer G, Meyer CHA, Richardson PL, Cruickshank J, Bartlett J. Cardiac sequelae of acute head injury. Br Heart 7 1982; 47: 221-6.

2 Lasky II, Nahum AM, Siegel AW. Cardiac injuries incurred by drivers in automobile accidents. 7 Forensic $S c i$ 1969; 14: 13-33.

3 Saunders CR, Doty DB. Myocardial contusion. Surg Gynecol Obstet 1977; 144: 595-603.

4 Demuth WE Jr, Zinsser HF Jr. Myocardial contusion. Arch Intern Med 1965; 115: 434-42.

5 Pearce W, Blair E. Significance of the electrocardiogram in heart contusion due to blunt trauma. F Trauma 1976; 16: $136-40$.

6 Lindsey D, Navin TR, Finley PR. Transient elevation of serum activity of $M B$ isoenzyme of creatine phosphokinase in drivers involved in automobile accidents. Chest 1978; 74: 15-8.

7 Reynolds M, Jones JW. CPK-MB isoenzyme determinations in blunt chest trauma. FACEP 1979; 8: 304-6.

8 Pomerantz M, Delgado F, Eiseman B. Unsuspected depressed cardiac output following blunt thoracic or abdominal trauma. Surgery 1971; 70: 865-71.

This letter was shown to Drs McLeod and Neil-Dwyer who reply as follows: 
Sir,

We are concerned that Larremore and Markovchick may do a disservice to those physicians and surgeons who manage cases of acute head injury if readers of this journal accept their contention that the cardiac sequelae of head injury are the result of only myocardial contusion. A large body of evidence, much of it referenced in our paper, indicates that catecholamine excess per se may cause myocardial damage. Many studies (also referenced in our paper and therefore not reiterated here) have shown that subarachnoid haemorrhage may have similar consequences. There can be no question of direct trauma in this instance. Your correspondents are in error when they suggest that prolonged coronary spasm or coronary occlusion is necessary for such damage to occur. The available evidence suggests that if the injury is a vascular one it is likely to be in the microvasculature. ${ }^{1}$ Platelet aggregation is one possible pathogenetic mechanism.

The paper by DeMuth and Zinsser ${ }^{2}$ quoted by Larremore and Markovchick does not state that focal myocardial necrosis is found in myocardial contusion; it emphasises that a discrete, sometimes extensive lesion, often resembling classical acute myocardial infarction is typically seen.

As emphasised in the methods section of our article, great care was taken to exclude conventional multisystem trauma patients and those who might have suffered steering wheel injuries. The seven cases reported are the result of almost two years' scrutiny of accident cases admitted through an accident and emergency department which has over 30000 new attendances a year.

We agree that treatment is currently unsatisfactory in this condition, but it would be wrong to necessarily apply rules for management of myocardial infarction in a situation where the pathophysiology may be very different. A more appropriate approach seems to be pharmacological antagonism of the catecholamine excess, either by adrenoceptor antagonists or possibly by calcium antagonists. Only when the underlying pathophysiology of a condition has been separated from a superficially similar problem (a good recent analogy of this might be right ventricular versus left ventricular myocardial infarction) can appropriate and perhaps very different treatment be given.

Andrew McLeod, Glen Neil-Dwyer, Box 3813, Division of Clinical Pharmacology, Duke University Medical Center, Durham, North Carolina 27710, USA.

\section{References}

1 Haft JI. Cardiovascular injury induced by sympathetic catecholamines. Prog Cardiovasc Dis 1974; 17: 73-86.

2 DeMuth WE Jr, Zinsser HF Jr. Myocardial contusion. Arch Intern Med 1965; 115: 434-42. 